\title{
The Developing Synapse: Construction and Modulation of Synaptic Structures and Circuits
}

\author{
Susana Cohen-Cory
}

\begin{abstract}
Synapse formation and stabilization in the vertebrate central nervous system is a dynamic process, requiring bi-directional communication between pre- and postsynaptic partners. Numerous mechanisms coordinate where and when synapses are made in the developing brain. This review discusses cellular and activity-dependent mechanisms that control the development of synaptic connectivity.
\end{abstract}

The function of the nervous system critically relies on the establishment of precise synaptic connections between neurons and specific target cells (Fig. 1). During synaptogenesis, synapses form, mature, and stabilize and are also eliminated by a process that requires intimate communication between pre- and postsynaptic partners. Most of our understanding of synapse formation and stabilization comes from extensive studies performed at the neuromuscular junction (NMJ). However, recent advances in methodologies that include real-time imaging of living neurons have provided insight into the molecular, cellular, and activity-dependent processes that guide synaptogenesis in the developing central nervous system (CNS). This review highlights several aspects of vertebrate synaptogenesis and its relation to activity-dependent processes, from the cellular mechanisms by which neurons communicate with each other to establish synaptic contacts to the role of activity during the development of topographically ordered neuronal maps. Emphasis is placed on the development of central excitatory synapses, and some aspects of NMJ development are also discussed.

\section{Synaptogenesis: A Microscopic View}

In the CNS, synapse assembly begins when axons approach their targets and establish contact with dendritic arbors or soma of their target neurons. Real-time imaging experiments demonstrate that both axonal and dendritic filopodia actively participate in synapse formation (Fig. 2). Highly dynamic interactions at contact sites of advancing axon growth cones and dendritic filopodia have been demonstrated in living zebrafish embryos in which pre- and postsynaptic partners were labeled with green fluorescent protein (GFP) (1). Highly motile dendritic filopodia in zebrafish embryos resemble those

Mental Retardation Research Center, Department of Psychiatry and Biobehavioral Sciences, University of California, Los Angeles, Los Angeles, CA 90095, USA. E-mail: scohenco@ucla.edu

Present address: Department of Neurology and Behavior, University of California, Irvine, Irvine, CA 92697, USA. of mammalian developing central neurons undergoing synaptogenesis both in culture $(2,3)$ and in vivo (4). Dynamic filopodia are also present in developing axon arbors before synapse differentiation (5-8) and have been implicated in synapse formation (9). Real-time imaging of GFP-labeled synaptic components and functional imaging of presynaptic sites (labeled with FM 1-43, a vital dye that reveals activityevoked synaptic vesicle recycling) have revealed the time course and sequence of events in CNS synaptogenesis. Imaging GFP-tagged synaptobrevin II (also known as VAMP II, a synaptic vesicle protein) in cultured hippocampal neurons revealed that transport packets containing preassembled synaptic vesicle components begin to accumulate at presynaptic sites immediately after axons and dendritic filopodia establish initial contact (10). Presynaptic components are assembled very rapidly, within 1 to 2 hours of initial contact between neurons (10, 11). Presynaptic differentiation is characterized by the appearance of varicosities containing accumulations of synaptic vesicles at the presynaptic side as well as by the onset of activityevoked vesicle recycling $(10,11)$. Real-time imaging has also revealed that rapid changes in postsynaptic structures are also necessary for synaptogenesis to be initiated $(1,2,4,12-14)$. The postsynaptic density protein 95 (PSD-95), the major component of the postsynaptic specialization at glutamatergic synapses, has served as a useful postsynaptic marker of developing synapses. Clustering of PSD-95 and glutamate receptor components at the postsynaptic site follows the functional and morphological differentiation of presynaptic structures in cultured hippocampal neurons $(11,13)$ but occurs simultaneously in cultured cortical neurons (9). Functional imaging of presynaptic sites (labeled with FM 1-43), coupled with PSD-95 retrospective immunocytochemistry (11) and dual color, simultaneous imaging of pre- and postsynaptic components (synaptophysin, a synaptic vesicle protein, and PSD-95) (13), revealed that presynaptic differentiation precedes postsynaptic differentiation in developing hippocampal neurons. Here, highly motile dendritic filopodia were progressively replaced by developing spines or protospines, which then matured into spines, tiny protrusions at which most excitatory synaptic contacts are made $(12,13)$. GFP-PSD-95 clusters were absent in filopodia but appeared coincident with filopodial differentiation into protospines and became more abundant in mature spines (12, 13), supporting the notion that filopodia and protospines are precursors to mature, functional glutamatergic synapses $(2,3,15)$. In cultured cortical neurons, however, glutamate receptorcontaining transport packets were rapidly recruited to axodendritic contact sites before PSD-95 recruitment, forming functional synapses along the dendrite length (9). Thus, experimental evidence indicates that rapid cellular and molecular events guide synaptogenesis in the CNS. However, further studies are necessary to clearly differentiate between events that guide the assembly of distinct synaptic circuits versus perceived differences that arise from limitations in existing methodologies (16).

The rapid dynamics of synapse assembly support the concept that synaptic remodeling may accompany the morphological maturation of axonal and dendritic arbors. Thereby, the dynamic growth of axonal and dendritic arbors may directly reflect the formation, stabilization, and elimination of synapses $(17,18)$. A correlate between synapse formation and dynamic remodeling of axon arbor structure was recently provided by studies using GFP-tagged synaptobrevin to visualize synapse dynamics in arborizing axons in vivo. Simultaneous imaging of GFP-synaptobrevin clusters within individual Xenopus optic axons delineated by a red fluorescent dye (DsRed or DiI) demonstrated that synaptogenesis is a dynamic process directly correlated to the active branching and remodeling of axon terminal arbors (8). Along the branching axon arbor more synapses were formed than eliminated, whereas a large proportion of synapses remained stable. Synapse formation in vivo occurs rapidly, in less than 2 hours (8), supporting earlier in vitro observations $(10,11)$. Thus, the active remodeling of synapses is closely correlated to the dynamic changes in axon arbor morphology, because axon arborization and synapse formation and elimination occur at similar rates $(6-8,19)$.

How is synapse formation related to axon arborization and arbor structure? Visualization of synaptic vesicle distribution along axon arbors in fixed tissues showed punctate synaptic protein localization along the entire extent of the arbor $(20,21)$, suggesting that synapses are evenly distributed along the axon arbor. Electron microscopy studies also revealed that ul- 
trastructurally identified synapses are localized throughout the axon arbor $(22,23)$, an observation recently confirmed by in vivo imaging studies (8). In more mature arbors, however, ultrastructurally identified synapses are preferentially localized to distal end branches (23). In vivo imaging studies further demonstrate that GFP-synaptobrevin-labeled synaptic sites are preferentially located at axon branching sites. In fact, time-lapse imaging revealed a new aspect in the relation between axon branching and synapse formation: Almost all new branches $(80.1 \pm 4.3 \%)$ originate at axon arbor sites rich in synaptic clusters (Fig. 3) (8). Consequently, synapse formation and stabilization may be required for new branch extension (24). The formation and stabilization of new synapses may, therefore, trigger the developmental, activitydependent increase in axon arbor complexity $(19,25,26)$.

During development, more synapses are established than ultimately will be retained. Therefore, the elimination of excess synaptic inputs is a critical step in synaptic circuit maturation. Synapse elimination is a competitive process that involves interactions between preand postsynaptic partners. The dynamics of synapse formation $(8,10,11)$ and of synapse elimination may be much more rapid in the CNS than at the NMJ, where synapse elimination has been well characterized. At the vertebrate NMJ, a single muscle cell is initially innervated by multiple motor axons. The transition from multiple innervation to innervation by a single motor axon occurs gradually as some terminal branches retract from each muscle fiber before others, a process requiring about 24 hours for withdrawal of the presynaptic terminal (27). Motor axons lose branches asynchronously and without spatial bias (28), suggesting that local interactions at each NMJ regulate synapse elimination. Synapse elimination at the NMJ is an activity-dependent process, where weakening of synaptic function is thought to precede synapse dismantling (29-31). Evidence from both the mammalian and Drosophila NMJ indicates that presynaptic disassembly precedes disassembly of the postsynaptic apparatus upon synapse destabilization $(28,32)$. In the CNS, as with the NMJ, a developmental, activity-dependent remodeling of synaptic circuits takes place by a process that may involve the selective stabilization of coactive inputs and the elimination of inputs with uncorrelated activity. The anatomical refinement of synaptic circuits occurs at the level of individual axons and dendrites (33-35) by a dynamic process that involves rapid elimination of synapses (36). As axons branch and remodel, synapses form and dismantle with synapse elimination occurring rapidly, in less than two hours (8). In vivo, the majority of branches destined to be eliminated do not express presynaptic markers before their retraction. This observation, together with the observation that most branches can establish synapses regardless of whether they are eventually stabilized or eliminated (8), supports the notion that rapid synapse disassembly precedes branch elimination. Correlating functional imaging of synaptic sites (FM 1-43 imaging) with presynaptic marker localization in culture hippocampal neurons in which glutamate receptor function was altered demonstrated that synapse disassembly in the CNS occurs rapidly, within 1.5 hours after synapses are no longer functional (37). Removal of presynaptic elements at central synapses thus occurs rapidly through an activity-dependent process, as demonstrated for the NMJ.

\section{Development of Synaptic Connectivity}

Many factors have been identified that influence synapse formation and refinement in both the NMJ and the CNS. Pre- and postsynaptic differentiation are coordinated by anterograde and retrograde interactions between the axon growth cone and the target cell. Activitydependent and -independent interactions guide the initial steps of synapse differentiation and formation. Neural activity may play permissive rather than instructive roles during some aspects of synaptogenesis. For example, at the $\mathrm{NMJ}, z$-agrin is a key molecule that coordinates postsynaptic differentiation. Motor neuron growth cones release $z$-agrin, a proteoglycan that induces clustering of acetylcholine receptors (AChRs) and other postsynaptic components on the muscle fiber surface. Agrin activates muscle-specific kinase (MuSK), a transmembrane receptor tyrosine kinase, and rapsyn, a membrane associated cytoplasmic protein also expressed in muscle (27). AChR clustering at the postsynaptic site is thought to be an activity-independent triggering event in neuromuscular synapse differentiation, because
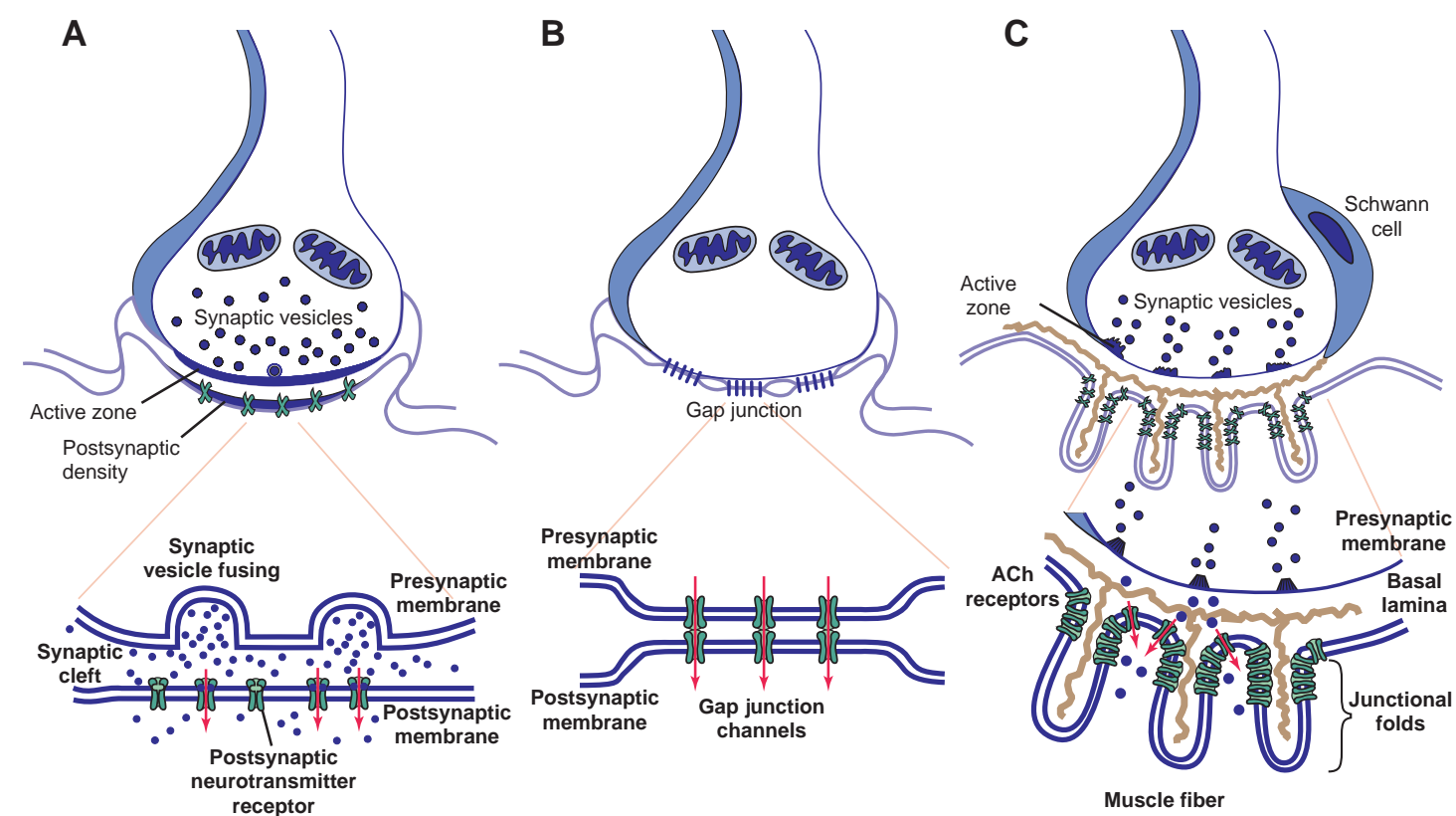

Fig. 1. Schematic representation of interneuronal and neuromuscular synapses. Synapses are asymmetric communication junctions formed between two neurons, or, at the neuromuscular junction (NMJ), between a neuron and a muscle cell. Chemical synapses enable cell-to-cell communication via secretion of neurotransmitters, whereas in less abundant electrical synapses signals are transmitted through gap junctions, specialized intercellular channels that permit ionic current flow. (A) At most interneuronal synapses, neurotransmitters are stored in synaptic vesicles and are released after synaptic vesicle fusion at the active zone (an event that is triggered by an action potential followed by a rapid influx of calcium into the presynaptic terminal). Neurotransmitter receptors and accessory molecules accumulate in the postsynaptic membrane directly opposite the active zone in a postsynaptic membrane specialization known as the postsynaptic density. (B) At electrical synapses, gap junctions between pre- and postsynaptic membranes permit current to flow passively through intercellular channels. In addition to ions, other molecules that modulate synaptic function (such as ATP and second messenger molecules) can diffuse through gap junctional pores. Electrical synapses synchronize electrical activity among populations of neurons. (C) At the mature NMJ, pre- and postsynaptic membranes are separated by a synaptic cleft containing extracellular proteins that form the basal lamina. Synaptic vesicles are clustered at the presynaptic release site, transmitter receptors are clustered in junctional folds at the postsynaptic membrane, and glial processes surround the nerve terminal. 
AChR clustering can occur at nerve-muscle contact sites in the presence of pharmacological activity blockers, as well as in uninnervated muscle fibers. Further, AChRs aggregate in muscle fibers of mice lacking all motor neuron innervation, in patterns that closely resemble those of innervated muscles $(38,39)$. AChR clustering is, however, dependent on MuSK expression by the postsynaptic muscle fiber $(38,39)$. Thus, initial phases of postsynaptic differentiation can occur in the absence of the nerve. Such activity-independent differentiation and patterning of signals within the muscle cell may serve to restrict synaptic innervation to the center of the muscle fiber and to selectively promote axonal innervation at specific sites (27). Though some aspects of postsynaptic differentiation may occur independently of the presynaptic motor neuron, selective stabilization of AChRs at synaptic sites requires neural-derived agrin, a process that is required for the activity-dependent maturation and stabilization of neuromuscular synapses (27).

Similar to the NMJ, neural activity may only be needed from some phases of synapse assembly in the CNS. Synapse assembly may occur in the absence of neurotransmission $(40,41)$. Genetic deletion of munc18-1, a neuron-specific protein essential for synaptic vesicle docking and neurotransmitter release from the presynaptic terminal, completely abolishes neurotransmitter secretion and synaptic transmission, yet apparently normal structural synapses and neuronal circuits form in these knock-out mice (40). Further, neurotransmitter receptor clustering at postsynaptic sites can occur under conditions of chronic receptor blockade (41), similarly suggesting that activity may not be required for initial aspects of synaptogenesis. However, numerous studies support the notion that some aspects of synapse formation and maturation require activity. Evidence that neurons are influenced by activity and secrete neurotransmitters at early stages of synaptogenesis supports a role for activity in early synapse assembly. Axon growth cones navigating toward their targets exhibit active synaptic vesicle cycles and upregulate neurotransmitter secretion once the growth cone approaches its target $(42,43)$. Additionally, neural activity modulates growth cone responses to repulsive and attractive guidance cues (44). The frequency and stability of initial axon-dendritic filopodial contacts may also be influenced by neuronal activity, because dendritic filopodial motility is modulated by depolarization-induced $\mathrm{Ca}^{2+}$ influx (1). In vivo, sensory deprivation that leads to reduction in activity can significantly influence dendritic filopodial and spine motility during the critical period of development, when developing neurons undergo synaptogenesis (4). Further support for a role for activity during initial synapse formation comes from studies showing that axonal and dendritic arborization are modulated by action potential and synaptic activity $(19,25,26,34,45-50)$. Blocking synaptic activity via the $N$-methyl-D-aspartate NMDA sub-type of glutamate receptors (NMDARs) inhibits dendritic arbor growth of immature Xenopus tectal neurons by preventing new branch addition $(19,48)$, suggesting that NMDAR activation is required for early synapse formation. A distinct, stabilizing role for glutamate receptor activation in the later phases of synaptogenesis and synapse maturation was suggested by studies where blocking NMDA or $\alpha$-amino-3-hydroxy-5methyl-4-isoxazoleproprionate (AMPA) receptor activity decreased dendritic arbor size in morphologically mature neurons (19, 34, 51). Metabotropic glutamate receptors may also participate in branch- and synapse-stabilizing responses (52). In contrast, chronic NMDAR blockade in developing hippocampal slice cultures increases synapse number during the early period of synaptogenesis (53). NMDAR-dependent changes resulted from increased dendritic num- ber and the corresponding increase in synapse number, without an increase in synapse density. Thus, experimental evidence supports a role for synaptic activity in the modulation of axonal and dendritic arbor complexity and, therefore, synaptic complexity. Further studies are required, however, to clearly distinguish how synaptic activity, through activation of distinct neurotransmitter receptors, differentially regulates synapse formation versus stabilization in distinct neuronal populations.

\section{Lessons from Synaptic Plasticity Models}

Most evidence demonstrating a role for synaptic activity in the formation and maturation of synapses comes from studies investigating synaptic structure and function in synaptic plasticity models. Similar to the NMJ, the accumulation of neurotransmitter receptors at the postsynaptic neuron is a key feature in the development and remodeling of central synapses. The synaptic content of distinct neurotransmitter receptor subunits and distinct receptor subtypes are differentially regulated during synapse formation and maturation. For example, at glutamatergic synapses, NMDARs are developmentally expressed before the expression of functional AMPA receptors (AMPARs). Activity-dependent mechanisms control postsynaptic levels of neurotransmitter receptors, with different mechanisms used for the synaptic targeting of NMDA and AMPA receptors $(41,54-$ 56). The translocation and clustering of NMDARs at synaptic sites is inversely modulated by NMDAR activity $(56,57)$. NMDAR activation also induces redistribution of AMPAR from intracellular pools to synaptic sites (58), and AMPAR levels are also dynamically regulated, with decreased activity increasing synaptic AMPAR content $(59,60)$. Rapid, activity-dependent synaptic recruitment of AMPARs is associated with the functional maturation of excitatory "silent" synapses, characterized by NMDAR currents but no AMPA currents (58). Interestingly, the developmental maturation of dendritic arbor morphology is correlated to the acquisition of AMPA synaptic

Fig. 2. Stages in the development of interneuronal synapses. (A and B) An axon growth cone approaches and interacts dynamically with a developing dendrite through a two-way filopodial communication. (C) The pre- and postsynaptic terminals form a morphologically unspecialized but functional contact. (D) Synaptic vesicles begin to accumulate at the presynaptic terminal, triggering neurotransmitter release and further synaptic differentiation. (E) Differentiation of the presynaptic terminal is followed by postsynaptic differentiation and by the accumulation of membrane components (such as PSD-95) at the postsynaptic side. (F) The recruitment of organizing molecules like PSD-95 at the postsynaptic specialization is followed by rapid neurotransmitter receptor accumulation at that site and by the functional maturation of the synapse. A spine synapse is used to illustrate the sequence of events during synapse development, but functional glutamatergic synapses can form along the dendrite shaft as well as in spineless dendrites. 
currents (34). Glutamate receptor expression is independently controlled at individual synaptic sites, and spine morphology is correlated with glutamate receptor function. Filopodia and developing spines do not express functional AMPA receptors, acquiring AMPAR function upon morphological maturation into a spine synapse (61). These observations further support the suggestion that active filopodia are precursors to the morphological equivalent of silent synapses that mature in an activity-dependent manner.

Studies investigating the effects of long-term synaptic plasticity have generally used experimental paradigms in which repetitive, high-frequency stimulation gives rise to synaptic potentiation [long-term potentiation (LTP)] that is accompanied by structural and molecular changes at the level of single synapses. Such LTP studies have revealed that NMDA-mediated synaptic activity triggers localized and rapid outgrowth of dendritic filopodia and new spine formation, morphological changes thought to correlate with the formation of new synapses $(14,62)$. Recent imaging experiments reveal that both NMDA and AMPA receptor activation are indeed involved in synapse formation and maturation. Visualizing GFP-tagged actin dynamics in cultured hippocampal neurons revealed that specific neuronal stimulation induces an active remodeling of pre- and postsynaptic actin at existing synaptic sites, an event that relates to synaptic vesicle fusion (63). Additionally, the appearance of new presynaptic actin puncta was observed upon high-frequency stimulation. F-actin is an essential component of developing synapses, and neuronal activity can redistribute and stabilize synaptic F-actin (64). In particular, NMDA and AMPA receptor blockers prevent presynaptic actin remodeling and the recruitment of new actin-labeled synaptic sites (63). Thus, specific glutamate receptor activation leads to new synapse formation, further indicating that morphological and functional changes go together during the activity-dependent formation, maturation, and stabilization of central synapses.

A number of cell adhesion molecules and tyrosine kinase receptor ligands have

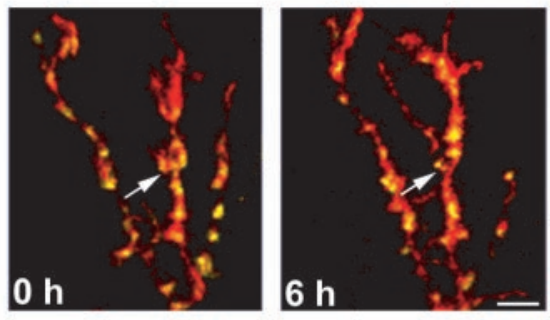

Neurotrophins as Synaptic Modulators

Neurotrophins, a family of neuronal growth factors that includes nerve growth factor (NGF), brain-derived neurotrophic factor (BDNF), neurotrophin 3 (NT-3), and neurotrophin $4 / 5$ (NT-4/5), are candidate molecules to modulate synaptogenesis in the developing brain. Though neurotrophins were initially characterized for their roles in promoting neuronal survival and differentiation, they also participate in many aspects of synapse development and function (74). Neurotrophins have emerged as key signals that mediate activity-dependent functional and structural plasticity in both the embryonic and mature brain (74-78). Neurotrophins may act as retrograde signals influencing presynaptic neurons, as well as anterograde factors acting on postsynaptic cells (79). Electrical activity and sensory input activity modulate both neurotrophin expression and re-

been implicated in modulating synaptogenesis largely by influencing synaptic function. Integrins are required for the functional maturation of hippocampal synapses in vitro because they mediate a switch in NMDAR subunit composition from an immature form (NR2B) to a mature form (NR2B-NR2A) (65). Cadherins have also been implicated in synaptic targeting and synapse formation, and their adhesive properties are themselves modulated by synaptic function (66-69). Neuroligins, a large group of transmembrane cell adhesion molecules enriched in the postsynaptic membrane of glutamatergic synapses, are strong candidate synaptogenic signals. Neuroligin-1 alone, expressed in nonneuronal cells, triggered presynaptic differentiation in contacting axons by binding to $\beta$-neurexins (70). The ephrins and their receptors, the Eph tyrosine kinases, participate in the activity-independent topographic organization of brain circuits (71) and may also participate in synapse formation and maturation by modulating NMDAR function $(72,73)$. Neurotrophins have been implicated in multiple aspects of synapse development and function, and evidence supporting their role in synaptogenesis is briefly discussed below. lease (80). The specific, activity-dependent vesicular release of BDNF from postsynaptic sites $(81)$ and the BDNF-induced protein synthesis in dendrites (82) suggest that neurotrophins can exert their effects at the level of individual synapses. Neurotrophin tyrosine kinase receptors themselves accumulate at synapses (both presynaptically and postsynaptically) $(83,84)$ with their activation $(85)$ and translocation to the membrane (86), depending on activity. Neurotrophins can profoundly influence axon and dendritic morphology and dendritic spine stability in developing neurons $(77,87)$, suggesting that they play important roles in the development of neuronal connectivity. In particular, BDNF has emerged as a key player in the development of neuronal connectivity. BDNF modulates axonal and dendritic branching and remodeling $(6,88-94)$, increases the efficacy of synaptic transmission (95-98), modulates the functional maturation of inhibitory and excitatory synapses (99-101), and is involved in the maturation and plasticity of neuronal networks $(78,102-104)$. Thus, BDNF may be required for synapse formation and stabilization $(17,74,75)$. Studies in transgenic mice demonstrate that target-derived BDNF regulates the expression of synaptic vesicle 
proteins and the density of synaptic innervation (105-110), suggesting that this neurotrophin can modulate synapses at multiple levels. For example, the number of innervating axons and the number of ultrastructurally identified synapses are reduced in the sympathetic system of BDNF knockout mice (105). Similarly, the number of axosomatic inhibitory synapses is significantly reduced in the cerebellum of conditional mutants lacking TrkB, the specific receptor for BDNF (109), and is increased upon TrkB activation (99). In addition, in vivo imaging experiments demonstrate that BDNF rapidly modulates synapse formation in arborizing axon terminals (8). Increasing BDNF levels at the target increases the number of GFP-synaptobrevin labeled presynaptic clusters in individual, arborizing Xenopus retinal axon terminals within 4 hours of treatment. In this system, BDNF modulates synapse number in two ways: It enhances axon arbor complexity, thus increasing total synaptic territory $(6,26)$, and it also increases synaptic innervation density per axon branch (8). Consequently, by modulating synapse number in addition to modulating axon arbor structure, BDNF is capable of influencing the functional complexity of neuronal circuits.

In addition to modulating synaptic architecture and number, BDNF modulates synapse maturation at multiple levels. At central glutamatergic synapses, BDNF promotes the transition of immature, "silent" synapses into mature AMPAR-containing synapses (111) by a mechanism that may regulate AMPAR expression (112). Neurotrophins can also modulate synaptic function by inducing presynaptic modifications in transmitter release $(80,95,98,113,114)$, although postsynaptic effects of BDNF have also been demonstrated (115). As demonstrated for central synapses, neurotrophins may influence synaptic function at the NMJ not only by modulating synaptic efficacy but also by modulating other aspects of synaptogenesis. In vivo alterations in muscle TrkB receptor signaling induce disassembly of postsynaptic specializations at the NMJ (106), suggesting BDNF and/or NT-4, another TrkB ligand, are involved in synapse maintenance. Recent studies provide a link between AChR synthesis, neurotrophin function, and activity-dependent changes in synaptic structure at the NMJ. Pharmacologic activity blockade during the early stages of synaptogenesis influences the organization of neuromuscular synapses by inducing excessive motor axon sprouting and preventing normal synapse elimination $(27,116)$. Activity blockade's influence on synaptic structure may result from interfering with neurotrophin action. Neurotrophins modulate neuregulin expression, a motor neuron-secreted growth factor that stimulates AChR synthesis in muscle fibers $(116,117)$. BDNF restores both neuregulin expression and synaptic structure in muscles deprived of synaptic activity (116). Thus, this evidence, together with observations that BDNF can prevent the destabilizing effects of activity blockade on actively arborizing axon terminals in the brain (26), provides a morphological link between neurotrophins and activity-dependent modulation of synapse formation.

Together, neurotrophins are potent morphogenetic modulators that can modify the structure of existing synapses and induce the formation of new synaptic contacts. The neurotrophin-induced morphological changes at the synapse may be intimately linked to their modulation of synaptic function, perhaps
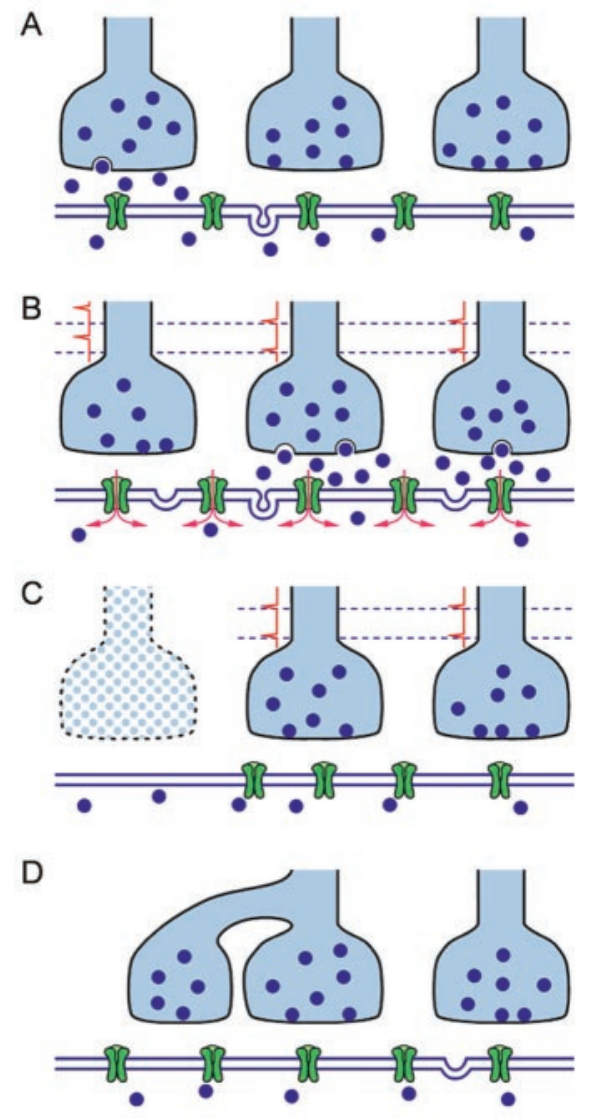

Fig. 4. Activity-dependent refinement of synaptic connections. (A) Synapses made by terminals of different axons co-innervating the same postsynaptic dendrite are initially maintained by low-level constitutive secretion of neurotransmitters. (B) Synchronous firing of two axon terminals (middle and right) leads to increased postsynaptic depolarization and neurotransmitter receptor activation (red arrows). (C) The unsynchronized axon terminal (left) does not experience postsynaptic spiking at the time of synaptic activation, therefore potentially reducing its retrograde neurotrophic support and ultimately resulting in the weakening of the synapse and the eventual withdrawal of the synaptic terminal. (D) Terminals that are active receive neurotrophic support and sprout (left) to establish new synapses. through the activation of common intracellular signaling pathways. The effects of neurotrophins on synapse number, synaptic efficacy, and neuronal morphology therefore support their role as activity-dependent modulators of synaptic structures and circuits. Whether neurotrophins are indeed the object of competition during the activity-dependent refinement of neuronal maps as it was originally proposed $(17,74,75,102)$ (Fig. 4) remains an open question.

\section{Establishment and Refinement of Neuronal Maps}

The vertebrate visual system has been extensively used as a model of synaptic competition during topographic map development. In mammals, retinal ganglion cells from each eye project contralaterally and ipsilaterally to the superior colliculus and the lateral geniculate nucleus (LGN). Within the LGN, the axonal projections from the two eyes are initially intermingled, then segregate into eye-specific layers during early development (18). In turn, the LGN inputs to the cortex segregate into eye-specific domains within cortical layer IV $(17,18)$. Alternate eye-specific stripes constitute the structural basis for the functionally defined system of ocular dominance columns that span all cortical areas. Neural activity modulates the early development of topographic visual maps by refining an initially imprecise and overlapping projection into eye-specific domains. An activity-dependent competitive process is thought to drive synapse and axon arbor pruning and, therefore, map refinement into eye-specific ocular dominance columns (17, 118). This view was recently challenged by evidence demonstrating that in the cat ocular dominance columns form before eye opening, shortly after LGN axons innervate the cortex (119-121). Direct anterograde tracer injections-rather than transneuronal transport of label from eye injections-showed that thalamocortical axons are already segregated into ocular dominance stripes before the onset of the critical period $(119,120)$. This segregation of thalamocortical inputs in layer IV of the visual cortex can take place in the absence of visually driven activity (120, 121). An activity-independent molecular mechanism may account for the early segregation of visual inputs (122). Thus, neuronal activity may exert a permissive rather than instructive role during the initial development of visual connectivity. However, patterned activity rather than the total amount of activity appears to be key in modulating synaptic competition and pruning (Fig. 4). In the retina, waves of correlated activity propagate through a synaptic network of amacrine and ganglion cells (123) and are synchronized by gap junctional communication between neurons $(124,125)$ before photoreceptors differ- 
entiate and visual input into the retina can be established. Blockade of neuronal activity in the retina or LGN prevents the axons from the two eyes from segregating into eye-specific layers (126-128). Similarly, increasing the frequency of retinal waves in one eye creates an imbalance in the competitive inputs and prevents the normal segregation of axons within the LGN (129). Thus, patterned neuronal activity is required for eye-specific segregation of inputs within the LGN, but whether activity-dependent competition is also responsible for the initial targeting of LGN inputs to the cortex [rather than molecular guidance cues (122)] or to maintain and refine a molecularly driven projection is not yet clear. At the NMJ, as occurs in the development of the visual system, synaptic inputs are temporally correlated before synapse refinement (130). At neuromuscular synapses, a competitive transition from multiple to single innervation occurs during early development that is thought to be modulated by the relative patterns of activity in the motor inputs innervating the same muscle fiber (27). Single motor neuron activity recordings from awake, behaving neonatal mice demonstrated that motor neuron activity is low but temporally correlated before the onset of synaptic competition (130). Thus, low-level activity may become asynchronous at the time that competition occurs, allowing the muscle fiber to detect the relative differences in the activity of its multiple motor inputs, triggering synapse elimination. Consequently, waves of correlated activity that spread among developing neurons through chemical synapses and/or gap junctions may contribute to early development and refinement of topographically ordered neuronal maps $(118,125)$.

Because of its remarkable plasticity, the visual system of nonmammalian vertebrates has been used as a model to explore the mechanisms by which neuronal activity modulates the development and refinement of topographic maps (131-133). In frogs and fish, each retina projects to the contralateral side of the optic tectum in a topographically organized manner. An inverted topographic map is initially formed by patterned molecular cues that guide axons to their targets $(71,131)$. Zebrafish embryo studies support the notion that the maturation and refinement of topographic maps involves activity-dependent processes. Mutants that have a temporally regulated reduction in sodium currents at the time that the retinotectal projection refines show significant dispersed projection patterns but minimal alterations in individual optic axon arbor morphologies (134). The alteration in the projection pattern occurs after the initial map is formed, suggesting that neuronal activity is necessary for synapse stabilization and refinement but not for the establishment of the topographic map. These studies, though supportive of the activity-indepen- dent view of topographic map formation (122), do not yet conclusively demonstrate that molecular cues alone are responsible for the initial establishment of topographic maps because sodium current function in the zebrafish mutants is developmentally downregulated. The classic observation that eye-specific stripes form in the optic tectum of frogs in which a single optic tectum is innervated by two eyes $(45,135)$ supports the hypothesis that activity plays a role in the establishment of topographic maps and that ocular dominance columns may arise by the activity-dependent sorting of eye-specific inputs. Experiments in ferrets with retinal projections redirected to the auditory pathway also support an early role for activity-dependent competition during synaptic circuit formation. In the rewired ferrets, retinal axons from the two eyes initially overlap within the auditory thalamus but later segregate into eye-specific clusters and form a topographically ordered, functional retinotopic map at an inappropriate target (136). Because topographic, molecular cues are organized differentially in distinct thalamocortical sensory circuits (auditory versus visual), the early rewiring data argue in favor of patterned activity playing an instructive role in the establishment of topographic maps. Careful analysis of the topographic organization of the somatosensory cortex of mutant mice lacking functional NMDA receptors (137) also supports the notion that neuronal activity is important for both the establishment and refinement of topographic maps (138).

\section{Conclusion}

Experimental evidence argues for both activityindependent and activity-dependent processes in regulating early synaptogenic events in the developing brain, from the level of individual synapses to the level of topographically organized neuronal maps. Though a role for activity in synapse maintenance and remodeling is clear, an instructive role for activity in early stages of synapse formation is less certain. Combination of electrophysiological, molecular, and imaging techniques has begun to shed light on the processes that control synaptogenesis at individual synapses and promises to provide novel insights on the cellular, molecular, and activity-dependent mechanisms that direct synaptogenesis at multiple levels within the developing brain.

\section{References and Notes}

1. J. D. Jontes, J. Buchanan, S. J. Smith, Nature Neurosci. 3, 231 (2000).

2. M. E. Dailey, S. J. Smith, J. Neurosci. 16, 2983 (1996)

3. N. E. Ziv, S. J. Smith, Neuron 17, 91 (1996).

4. B. Lendvai, E. A. Stern, B. Chen, K. Svoboda, Nature 404, 876 (2000).

5. N. A. O'Rourke, S. E. Fraser, Neuron 5, 159 (1990). 6. S. Cohen-Cory, S. E. Fraser, Nature 378, 192 (1995)
7. S. Witte, H. Stier, H. T. Cline, J. Neurobiol. 31, 219 (1996).

8. B. Alsina, T. Vu, S. Cohen-Cory, Nature Neurosci. 4 1093 (2001).

9. P. Washbourne, J. E. Bennett, A. K. McAllister, Nature Neurosci. 5, 751 (2002).

10. S. E. Ahmari, J. Buchanan, S. J. Smith, Nature Neurosci. 3, 445 (2000).

11. H. V. Friedman, T. Bresler, C. C. Garner, N. E. Ziv, Neuron 27, 57 (2000).

12. G. S. Marrs, S. H. Green, M. E. Dailey, Nature Neurosci. 4, 1006 (2001).

13. S. Okabe, A. Miwa, H. Okado, J. Neurosci. 21, 6105 (2001).

14. M. Maletic-Savatic, R. Malinow, K. Svoboda, Science 283, 1923 (1999).

15. J. D. Jontes, S. J. Smith, Neuron 27, 11 (2000).

16. S. E. Ahmari, S. J. Smith, Neuron 34, 333 (2002).

17. L. C. Katz, C. J. Shatz, Science 274, 1133 (1996).

18. C. J. Shatz, Neuron 5, 745 (1990).

19. I. Rajan, S. Witte, H. T. Cline, J. Neurobiol. 38, 357 (1999).

20. M. A. Silver, M. P. Stryker, J. Neurosci. 19, 10829 (1999).

21. E. M. Pinches, H. T. Cline, J. Neurobiol. 35, 426 (1998).

22. G. Campbell, C. J. Shatz, J. Neurosci. 12, 1847 (1992).

23. L. Yen, J. T. Sibley, M. Constantine-Paton, J. Neurosci. 15, 4712 (1995).

24. J. E. Vaughn, Synapse 3, 255 (1989).

25. A. Antonini, M. P. Stryker, J. Comp. Neurol. 369, 64 (1996).

26. S. Cohen-Cory, J. Neurosci. 19, 9996 (1999).

27. J. R. Sanes, J. W. Lichtman, Nature Rev. Neurosci. 2, 791 (2001).

28. C. R. Keller-Peck et al., Neuron 31, 381 (2001).

29. R. J. Balice-Gordon, C. K. Chua, C. C. Nelson, J. W. Lichtman, Neuron 11, 801 (1993).

30. R. J. Balice-Gordon, J. W. Lichtman, Nature 372, 519 (1994).

31. H. Colman, J. Nabekura, J. W. Lichtman, Science 275, 356 (1997).

32. B. A. Eaton, R. D. Fetter, G. W. Davis, Neuron $\mathbf{3 4}, 729$ (2002).

33. A. Antonini, M. P. Stryker, Science 260, 1819 (1993).

34. H. T. Cline, Curr. Opin. Neurobiol. 11, 118 (2001).

35. W. T. Wong, R. O. Wong, Curr. Opin. Neurobiol. 10, 118 (2000).

36. J. T. Trachtenberg, M. P. Stryker, J. Neurosci. 21, 3476 (2001)

37. F. W. Hopf, J. Waters, S. Mehta, S. J. Smith, J. Neurosci. 22, 775 (2002).

38. X. Yang et al., Neuron 30, 399 (2001).

39. W. Lin et al., Nature 410, 1057 (2001).

40. M. Verhage et al., Science 287, 864 (2000).

41. A. M. Craig, H. Boudin, Nature Neurosci. 4, 569 (2001).

42. J. Roos, R. B. Kelly, Nature Neurosci. 3, 415 (2000).

43. Z. P. Xie, M. M. Poo, Proc. Natl. Acad. Sci. U.S.A. 83, 7069 (1986)

44. G. Ming, J. Henley, M. Tessier-Lavigne, H. Song, M. Poo, Neuron 29, 441 (2001).

45. T. A. Reh, M. Constantine-Paton, J. Neurosci. 5, 1132 (1985).

46. D. W. Sretavan, C. J. Shatz, M. P. Stryker, Nature 336, 468 (1988).

47. A. Antonini, M. P. Stryker, J. Neurosci. 13, 3549 (1993). 48. I. Rajan, H. T. Cline, J. Neurosci. 18, 7836 (1998).

49. G. Y. Wu, H. T. Cline, Science 279, 222 (1998).

50. W. T. Wong, R. O. Wong, Nature Neurosci. 4, 351 (2001).

51. D. J. Zou, H. T. Cline, J. Neurosci. 19, 8909 (1999).

52. F. Miskevich, W. Lu, S. Y. Lin, M. Constantine-Paton, J. Neurosci. 22, 226 (2002).

53. A. Luthi, L. Schwyzer, J. M. Mateos, B. H. Gahwiler, R. A. McKinney, Nature Neurosci. 4, 1102 (2001).

54. S. H. Lee, M. Sheng, Curr. Opin. Neurobiol. 10, 125 (2000).

55. M. Sheng, S. H. Lee, Cell 105, 825 (2001).

56. M. Sheng, Proc. Natl. Acad. Sci. U.S.A. 98, 7058 (2001).

57. A. Rao, A. M. Craig, Neuron 19, 801 (1997).

58. S. H. Shi et al., Science 284, 1811 (1999).

59. D. V. Lissin et al., Proc. Natl. Acad. Sci. U.S.A. 95 7097 (1998).

60. R. J. O'Brien et al., Neuron 21, 1067 (1998). 
61. M. Matsuzaki et al., Nature Neurosci. 4, 1086 (2001). 62. F. Engert, T. Bonhoeffer, Nature 399, 66 (1999).

63. M. A. Colicos, B. E. Collins, M. J. Sailor, Y. Goda, Cell 107, 605 (2001)

64. W. Zhang, D. L. Benson, J. Neurosci. 21, 5169 (2001).

65. P. Chavis, G. Westbrook, Nature 411, 317 (2001).

66. H. Tanaka et al., Neuron 25, 93 (2000).

67. J. L. Bruses, Curr. Opin. Cell. Biol. 12, 593 (2000)

68. B. Ranscht, Int. J. Dev. Neurosci. 18, 643 (2000)

69. O. Bozdagi, W. Shan, H. Tanaka, D. L. Benson, G. W. Huntley, Neuron 28, 245 (2000).

70. P. Scheiffele, J. Fan, J. Choih, R. Fetter, T. Serafini, Cell 101, 657 (2000)

71. D. D. O'Leary, D. G. Wilkinson, Curr. Opin. Neurobiol. 9, 65 (1999).

72. M. B. Dalva et al., Cell 103, 945 (2000)

73. M. A. Takasu, M. B. Dalva, R. E. Zigmond, M. E. Greenberg, Science 295, 491 (2002).

74. M. M. Poo, Nature Rev. Neurosci. 2, 24 (2001).

75. W. D. Snider, J. W. Lichtman, Mol. Cell. Neurosci. 7 , 433 (1996).

76. E. M. Schuman, Curr. Opin. Neurobiol. 9, 105 (1999).

77. A. K. McAllister, L. C. Katz, D. C. Lo, Annu. Rev. Neurosci. 22, 295 (1999).

78. N. Prakash, S. Cohen-Cory, R. D. Frostig, Nature $\mathbf{3 8 1}$ 702 (1996).

79. C. S. von Bartheld, X. Wang, R. Butowt, Mol. Neurobiol. 24, 1 (2001).

80. A. F. Schinder, M. Poo, Trends. Neurosci. 23, 639 (2000).

81. M. Hartmann, R. Heumann, V. Lessmann, EMBO J. 20, 5887 (2001).

82. G. Aakalu, W. B. Smith, N. Nguyen, C. Jiang, E. M. Schuman, Neuron 30, 489 (2001)

83. C. Aoki et al., J. Neurosci. Res. 59, 454 (2000).

84. K. Wu et al., Brain. Res. Mol. Brain. Res. 43, 286 (1996).

85. R. Aloyz, J. P. Fawcett, D. R. Kaplan, R. A. Murphy, F. D. Miller, Learn. Mem. 6, 216 (1999).

86. A. Meyer-Franke et al., Neuron 21, 681 (1998).

87. A. K. McAllister, Cereb. Cortex. 10, 963 (2000).

88. , D. C. Lo, L. C. Katz, Neuron 15, 791 (1995)

89. B. Lom, S. Cohen-Cory, J. Neurosci. 19, 9928 (1999).

90. A. K. McAllister, L. C. Katz, D. C. Lo, Neuron 17, 1057 (1996).
91. H. W. Horch, A. Kruttgen, S. D. Portbury, L. C. Katz Neuron 23, 353 (1999).

92. T. A. Yacoubian, D. C. Lo, Nature Neurosci. 3, 342 (2000).

93. A. Inoue, J. R. Sanes, Science 276, 1428 (1997).

94. A. Shimada, C. A. Mason, M. E. Morrison, J. Neurosci. 18, 8559 (1998).

95. L. Boulanger, M. M. Poo, Nature Neurosci. 2, 346 (1999).

96. K. W. Kafitz, C. R. Rose, H. Thoenen, A. Konnerth, Nature 401, 918 (1999).

97. H. Kang, E. M. Schuman, Science 267, 1658 (1995).

98. A. M. Lohof, N. Y. Ip, M. M. Poo, Nature 363, 350 (1993).

99. F. J. Seil, R. Drake-Baumann, J. Neurosci. 20, 5367 (2000).

100. C. Vicario-Abejon, C. Collin, R. D. McKay, M. Segal, J. Neurosci. 18, 7256 (1998).

101. L. C. Rutherford, S. B. Nelson, G. G. Turrigiano, Neuron 21, 521 (1998).

102. R. J. Cabelli, D. L. Shelton, R. A. Segal, C. J. Shatz, Neuron 19, 63 (1997)

103. J. L. Hanover, Z. J. Huang, S. Tonegawa, M. P. Stryker, J. Neurosci. 19, RC40 (1999).

104. B. Xu et al., Neuron 26, 233 (2000).

105. C. G. Causing et al., Neuron 18, 257 (1997).

106. M. Gonzalez et al., Neuron 24, 567 (1999).

107. A. Martinez et al., J. Neurosci. 18, 7336 (1998).

108. L. D. Pozzo-Miller et al., J. Neurosci. 19, 4972 (1999).

109. B. Rico, B. Xu, L. F. Reichardt, Nature Neurosci. 5 225 (2002)

110. W. J. Tyler, L. D. Pozzo-Miller, J. Neurosci. 21, 4249 (2001).

111. C. Itami, K. Mizuno, T. Kohno, S. Nakamura, Brain Res. 857, 141 (2000).

112. M. Narisawa-Saito, J. Carnahan, K. Araki, T. Yamaguchi, H. Nawa, Neuroscience 88, 1009 (1999).

113. A. F. Schinder, B. Berninger, M. Poo, Neuron 25, 151 (2000).

114. X. H. Wang, M. M. Poo, Neuron 19, 825 (1997)

115. Y. Kovalchuk, E. Hanse, K. W. Kafitz, A. Konnerth, Science 295, 1729 (2002)
116. J. A. Loeb, A. Hmadcha, G. D. Fischbach, S. J. Land, V. L. Zakarian, J. Neurosci. 22, 2206 (2002).

117. J. A. Loeb, G. D. Fischbach, J. Neurosci. 17, 1416 (1997)

118. M. B. Feller, Neuron 22, 653 (1999).

119. J. C. Crowley, L. C. Katz, Nature Neurosci. 2, 1125 (1999).

120. Science 290, 1321 (2000).

121. M. C. Crair, D. C. Gillespie, M. P. Stryker, Science 279, 566 (1998).

122. J. C. Crowley, L. C. Katz, Curr. Opin. Neurobiol. 12, 104 (2002).

123. M. B. Feller, D. P. Wellis, D. Stellwagen, F. S. Werblin, C. J. Shatz, Science 272, 1182 (1996).

124. W. T. Wong, J. R. Sanes, R. O. Wong, J. Neurosci. 18 8839 (1998).

125. R. O. Wong, Annu. Rev. Neurosci. 22, 29 (1999).

126. A. A. Penn, P. A. Riquelme, M. B. Feller, C. J. Shatz, Science 279, 2108 (1998)

127. D. W. Sretavan, C. J. Shatz, J. Neurosci. 6, 990 (1986).

128. C. J. Shatz, M. P. Stryker, Science 242, 87 (1988).

129. D. Stellwagen, C. J. Shatz, Neuron 33, 357 (2002)

130. K. E. Personius, R. J. Balice-Gordon, Neuron 31, 395 (2001)

131. R. W. Sperry, Proc. Nat. Acad. Sci. U.S.A. 50, 703 (1963)

132. H. T. Cline, Trends Neurosci. 14, 104 (1991).

133. E. A. Debski, H. T. Cline, Curr. Opin. Neurobiol. 12 93 (2002)

134. L. Gnuegge, S. Schmid, S. C. Neuhauss, J. Neurosci. 21, 3542 (2001)

135. M. Constantine-Paton, H. T. Cline, E. Debski, Annu. Rev. Neurosci. 13, 129 (1990)

136. M. Sur, C. A. Leamey, Nature Rev. Neurosci. 2, 251 (2001).

137. T. Iwasato et al., Nature 406, 726 (2000).

138. J. H. Kaas, K. C. Catania, Bioessays 24, 334 (2002).

139. I thank R. Frostig, B. Lom, B. Alsina, and C. Colwell for discussions and comments; B. Hu for his contribution to Fig. 3; and D. Crandall for assistance with graphic art. S.C.C. has been funded by awards from the Alfred P. Sloan, Stein-Oppenheimer, and Arnold and Mabel Beckman Foundations and by the $\mathrm{Na}$ tional Eye Institute.

\title{
RE V I E W
}

\section{Postsynaptic Signaling and Plasticity Mechanisms}

\author{
Morgan Sheng* and Myung Jong Kim
}

\begin{abstract}
In excitatory synapses of the brain, specific receptors in the postsynaptic membrane lie ready to respond to the release of the neurotransmitter glutamate from the presynaptic terminal. Upon stimulation, these glutamate receptors activate multiple biochemical pathways that transduce signals into the postsynaptic neuron. Different kinds of synaptic activity elicit different patterns of postsynaptic signals that lead to short- or long-lasting strengthening or weakening of synaptic transmission. The complex molecular mechanisms that underlie postsynaptic signaling and plasticity are beginning to emerge.
\end{abstract}

Excitatory synapses of the brain primarily use glutamate as their neurotransmitter. Different classes of glutamate receptors in the postsynap-

Picower Center for Learning and Memory, RIKEN-MIT Neuroscience Research Center, Howard Hughes Medical Institute, Massachusetts Institute of Technology, Cambridge, MA 02139, USA.

*To whom correspondence should be addressed. Email: msheng@mit.edu tic membrane transduce the glutamate signal released from the presynaptic terminal into electrical and biochemical events in the postsynaptic neuron. The $\alpha$-amino-3-hydroxy-5-methyl-4isoxazolepropionic acid (AMPA)-type glutamate receptor opens in response to glutamate binding and mediates most of the rapid excitatory postsynaptic current (EPSC). The $N$-methyl-D-aspartate (NMDA)-type glutamate receptor is calcium-permeable and opens in response to glutamate only when the postsynaptic membrane is concomitantly depolarized. Different patterns of activation of NMDA receptors (NMDARs) can lead to either long-term potentiation (LTP) or long-term depression (LTD) of synaptic strength. These long-lasting forms of synaptic plasticity are intensively studied because they may represent ways of encoding "memories" in the brain.

Changes in synaptic strength can occur by presynaptic mechanisms such as altered neurotransmitter release (1-3). Recent evidence, however, also points to a postsynaptic locus for the expression of plasticity, in which changing the activity and/or abundance of postsynaptic AMPA receptors (AMPARs) is the primary means of modulating synaptic transmission. Wherever the site of plasticity expression, there is general agreement that the initiating events ("induction") of LTP and 